УДК 349.7

\title{
ЛИЗИКОВА М.С. \\ ОБЕСПЕЧЕНИЕ БЕЗОПАСНОСТИ В ОБЛАСТИ ИСПОЛЬЗОВАНИЯ АТОМНОЙ ЭНЕРГИИ В УСЛОВИЯХ ПАНДЕМИИ: ПРАВОВЫЕ АСПЕКТЫ
}

\begin{abstract}
Ключевые слова: ядерная безопасность, регулирование ядерной безопасности, МАГАТЭ, МЭА, рекомендации МАГАТЭ, атомная энергетика, АЭС, использование атомной энергии, пандемия, чрезвычайные ситуации, коронавирус, COVID-19.

В статье поставлена проблема обеспечения безопасности в области использования атомной энергии в условиях пандемии. На основе анализа мер по обеспечению безопасной эксплуатации АЭС, принятых национальными регулирующими организациями в этот период, а также деятельности МАГАТЭ и иных международных организаций по оказанию помощи операторам АЭС и обмену опытом по смягчению воздействия пандемии на атомную отрасль, сделаны выводы о необходимости всестороннего исследования поставленной проблемы, извлечения «уроков» пандемии для атомной энергетики, многостороннего сотрудничества для сдерживания пандемии и смягчения ее последствий.
\end{abstract}

LIZIKOVA, M.S.

SECURITY IN THE FIELD OF USE OF ATOMIC ENERGY IN THE CONDITIONS OF PANDEMIA: LEGAL ASPECTS

Keywords: nuclear safety, nuclear safety regulation, IAEA, IEA, IAEA recommendations, nuclear energy, nuclear power plants, atomic energy use, pandemic, emergencies, coronavirus, COVID-19.

In the article poses the problem of ensuring safety in the field of the use of atomic energy in the conditions of pandemia. Based on an analysis of measures taken by national regulatory organizations to ensure the safe operation of nuclear power plants during this period, as well as the activities of the IAEA and other international organizations to provide assistance to nuclear power plant operators and exchange experience on mitigating the impact of a pandemic on the nuclear industry and minimizing its consequences, it concluded on the necessity of comprehensive study of the problem posed, the lessons learned from the pandemic for nuclear energy, and multilateral cooperation to contain the pandemic and mitigate its consequences.

Пандемия короновируса (COVID-19), начавшаяся в конце 2019 года, оказала значительное воздействие на все сферы экономики, в том числе и на глобальный энергетический сектор. В то время как некоторые отрасли оказались в кризисной ситуации или вовсе остановились, последний проявляет устойчивость, сохраняя способность бесперебойно вырабатывать электроэнергию, необходимую как для жизнеобеспечения во время пандемии, так и для борьбы с ней.

В то же время, по данным Международного энергетического агентства (МЭА), опубликованным в отчете Global Energy Review 2020 [1], результатом влияния пандемии явилось «ошеломительное» [2] снижение мирового спроса на энергию в первом квартале 2020 года (сравнению с аналогичным периодом 2019 года) на 3,8\% и прогноз его дальнейшего падения до $5 \%$. Сильнее всего это ощутили угольная, нефтяная и газовая отрасли. Не стала исключением и атомная энергетика. Несмотря на продолжение работы АЭС, объем производства атомной энергии в первом квартале 2020 г. снизился на $3 \%$, а к концу года отрасль ожидает сокращение показателей более чем на $1 \%$. Однако причинами этого, по оценке МЭА, являются не только вызванные пандемией падение спроса на энергию, задержки с плановым обслуживанием АЭС и задержки строительства нескольких новых ректоров, но также мягкая погода и вывод некоторых реакторов из эксплуатации. Сокращение производства атомной энергии наблюдается в Европе и США, в то время как Китай, запустивший два новых реактора, и Россия, окончившая ремонт двух энергоблоков, напротив, показывают его рост [3, 4].

Не следует недооценивать роль, которую во многих странах играет атомная энергетика в обеспечении бесперебойного снабжения электроэнергией в период кризиса, вызванного COVID19. Во Франции, Германии, Бельгии, Швеции и др. она, работая в режиме следования за нагрузкой, обеспечила энергосистеме гибкость и, соответственно, безопасность. Кроме того, атомная отрасль обладает возможностью сыграть еще большую роль в борьбе с COVID-19, оказывая помощь системе здравоохранения. Так, например, ГК «Росатом» осуществляет стерилизацию медицинской продукции - защитных масок и медицинских транспортных систем, используемых для тестирования на наличие вируса COVID-19 [5], действующая в Канаде программа модернизации и продления срока службы АЭС «Брюс» предусматривает изучение новых областей применения радиоизотопов в ядерной медицине, в том числе для производства медицинского оборудования и средств индивидуальной защиты медицинских работников [6].

Будучи стратегически важной отраслью, атомная энергетика, в свою очередь, нуждается в защите от угрозы COVID-19 и минимизации его последствий для нее. Сохранение здоровья высококвалифицированных кадров, чрезвычайные меры реагирования, принимаемые 


\section{ПРАВО}

национальными операторами АЭС, обеспечение непрерывной эксплуатации ядерных реакторов, принятие решений о сокращении или приостановке не имеющих жизненно важного значения для обеспечения непрерывной эксплуатации АЭС операций, сокращение или прекращение объемов добычи урана и вероятность сбоя поставок, перенос сроков технического обслуживания объектов и загрузки нового топлива, сокращение численности персонала, осуществляющего эти работы, внедрение новых методов работы, в том числе и при строительстве АЭС, повышение риска террористических актов и кибератак - далеко не полный перечень вызовов COVID-19, которые в настоящее время еще только предстоит преодолеть, а влияние и последствия их на обеспечение безопасности в данной сфере - оценить в будущем. Это позволяет уже сегодня говорить о появлении такой серьезной проблемы как обеспечение безопасности в области использования атомной энергии в условиях пандемии и о необходимости ее всестороннего исследования, в том числе в правовом аспекте.

Проблема влияния пандемии на обеспечение безопасности в области использования атомной энергии была поднята в США в 2006 году в рамках семинара «Поддержание безопасных ядерных операций во время пандемии гриппа» [7], проведенного Комиссией по ядерному регулированию (NRC). В числе наиболее актуальных вопросов были вопросы о потенциальной необходимости изолировать работников на ранних стадиях вспышки заболевания и об оценке влияния высоких показателей невыхода на работу.

В 2007 году Институт ядерной энергии (NEI), представил на рассмотрение Комиссии по ядерному регулированию (NRC) проект «Pandemic Licensing Plan» [8]. В нем, на основе предположения вероятности сокращения численности персонала АЭС ниже уровней, необходимых для поддержания полного соответствия всем нормативным требованиям национального регулятора, в результате пандемии гриппа и прогноза по регламентации деятельности, необходимой для продолжения работы с сокращением штатного расписания в течении 4-6 недель, сделан вывод, что наиболее эффективным ответом на пандемию является введение «права на усмотрение».

Это право предоставило бы лицензиатам возможность переносить сроки отдельных видов деятельности, программных и административных требований, а также принимать компенсационные меры для смягчения последствий отступления от установленных требований при обязательном соблюдении норм здравоохранения и безопасности, а также протоколировании каждого несоответствия и обязательства их устранения во время или после пандемии.

При этом предлагаемый в проекте объем допустимой нормативной помощи варьировался, путем установления 3 категорий такой помощи, в зависимости от степени влияния на функциональность, связанную с безопасностью. Так как требования эксплуатации, связанные с безопасностью SSC, не могут быть поставлены под угрозу ни при каких обстоятельствах, соответственно, для этого вида требований нормативное облегчение или их минимизация не допускается. В иных случаях возможно частичное или существенное облегчение, объем которого целесообразно установить до начала пандемии. В связи с чем, NEI было рекомендовано Комиссии по ядерному регулированию: разработать и включить в действующие правила дополнительное руководство для расширения эксплуатационной гибкости, доступной лицензиатам во время пандемии; разработать с привлечением заинтересованных сторон временную политику защиты прав на усмотрение АЭС во время пандемии; заранее санкционировать усмотрение в отношении требований, которые не оказывают существенного влияния на функциональность, связанную с безопасностью SSC; размещать на веб-сайте информацию, связанную с утвержденными действиями по обеспечению соблюдения требований конкретных АЭС; определить нормативную базу управления чрезвычайными и неотложными изменениями технических спецификаций, связанных с пандемией и др. Согласно проекту, это бы уравновесило риск от продолжающейся работы с риском от региональных отключений и нестабильности энергосистемы.

Однако проект был отклонен по причине принципиального несогласия Комиссии с приведенным выше доводом, так как предлагаемое «нормативное освобождение» повлечет за собой повышение радиологического риска во время пандемии, а также необходимость решения ряда юридических вопросов.

Проблема сохранения здоровья персонала АЭС и других объектов отрасли с особой остротой встала во время вспышки COVID-19. Рекомендации по подготовке и реализации планов 


\section{ПРАВО}

реагирования на различные виды опасностей содержатся в разработанной МАГАТЭ серии «Руководство по промышленной безопасности ядерных объектов» [9]. На национальном уровне, как правило, пандемии охватываются мероприятиями по планированию действий в чрезвычайных ситуациях. Однако, как показала практика, они не рассчитаны на такие масштабы [10].

Меры, принимаемые национальными регулирующими организациями и направленные на сохранение здоровья, и защиту оперативного персонала от короновируса, различны. В их числе: изоляция ключевых специалистов, сокращение персонала, профилактический карантин, социальное дистанцирование, смещение смен или времени работы, ослабление мер контроля за режимом рабочего времени, перевод второстепенных сотрудников на удаленную работу, измерение температуры работников и тестирование на COVID-19 и др. - вплоть до прекращения работы и закрытия объекта.

Регламентация рабочего времени персонала АЭС и других объектов атомной энергетики осуществляется национальными регулирующими организациями. Однако такое внезапное и потенциально долгосрочное сокращение численности персонала в действовавших до начала пандемии нормативных актах не было предусмотрено. Поэтому конкретные меры и решения нередко принимались в «режиме реального времени».

Так, в США Комиссией по ядерному регулированию (NRC) было принято, отклоненное в 2007 году, решение предоставить лицензиатам гибкость в управлении кадровыми ресурсами для поддержания эксплуатационной безопасности и защиты АЭС в период, когда штатное расписание объекта может быть сокращено из-за COVID-19, при условии, что это безопасно и законно [11]. Для временного освобождения от ограничений рабочего времени предусмотрено направление письменного запроса по электронной почте. При этом сохраняется обязанность проводить оценку усталости, а также принимать альтернативные административные меры контроля за усталостью. В числе иных мер: создание специализированных Web-pecypсов для лицензиатов АЭС и общественности; возможность отложить выдачу некоторых счетов-фактур лицензиата; право требовать от инспекторов на АЭС и заводах по производству топлива дистанционной работы; перенос плановых проверок лицензиатов материалов, площадок для снятия с эксплуатации и автономных хранилищ отработавшего топлива; в том числе рассмотрения заявок и продлений лицензий, запросов на изменение лицензии, принудительного исполнения и т.п. в условиях удаленной работы. Более того, США, в случае недостаточного количества персонала, допускают закрытие любой АЭС на своей территории.

На всех российских АЭС ГК «Росатомом» был введен особый режим, а также разработан ряд дополнительных сценариев развития ситуации и мер по реагированию на любые случаи, связанные с возможными угрозами здоровью ключевого персонала АЭС [12]. Например, все сотрудники Белоярской АЭС, где один из работников заболел коронавирусом, за исключением оперативного персонала, были переведены на удаленную работу, в то время как последний переведен на проживание в профилакторий с полной изоляцией, круглосуточным медицинским наблюдением, дезинфекцией помещений и передвижением от профилактория до АЭС в автобусе, подвергаемом постоянной санобработке [13].

В ОАЭ, согласно принятому Федеральным управлением по ядерному регулированию (FANR) Плану управления непрерывностью бизнеса и создания целевой группы COVID-19 по антикризисному управлению [14], наряду с переводом сотрудников на удаленную работу и сокращением числа постоянных инспекторов на площадке АЭС в Бараке, осуществляется активный поиск инновационных способов удаленного проведения инспекционных и правоприменительных мероприятий с помощью цифровых средств.

За период пандемии COVID-19 ни одна из эксплуатируемых в мире АЭС не была закрыта в силу признания их основными службами, в то время как работа иных объектов была приостановлена. Например, урановый рудник Сигар-Лейк (Канада) на время пандемии COVID-19 перевели в режим безопасного ухода и технического обслуживания, также были закрыты шахты в ОАЭ, завод по переработке ядерного топлива в Селлафилде во Франции и др.

В условиях пандемии крайне важен обмен опытом по смягчению ее воздействия на атомную отрасль и минимизации последствий для последней. Это, в свою очередь, будет способствовать извлечению «уроков» необходимых для реагирования на подобные ситуации в будущем и выработке необходимых превентивных мер. Решение данной задачи взяли на себя 
международные организации, осуществляющие деятельность в области использования атомной энергии. В частности, сбор информации от государств-участников о принятых ими мерах по обеспечению наличия достаточного количества персонала для безопасной и надежной работы атомных электростанций МАГАТЭ, совместно с Агентством по ядерной энергии ОЭСР, осуществляют посредством онлайн-платформы IRS [15]. Для оказания помощи операторам АЭС в эксплуатации, техническом обслуживании, осуществлении перегрузки топлива и текущего ремонта в сложившейся ситуации МАГАТЭ была создана сеть «Опыт эксплуатации АЭС в условиях пандемии COVID-19» [16]. Выводы, сделанные в результате анализа полученной информации, должны найти место в соответствующих документах МАГАТЭ и, тем самым, способствовать укреплению режима ядерной безопасности.

О признании проблемы обеспечения безопасности в области использования атомной энергии в условиях пандемии и ее последствиях свидетельствует также вынесение ее обсуждения на международный уровень. Так, 19 мая 2020 года состоялся вебинар «Влияние пандемии COVID19 на атомную энергетику и необходимость инноваций» [17], в рамках которого представители МАГАТЭ, Всемирной ядерной ассоциации (WNA) и регулирующих органов отдельных государств обсудили вопросы эффективности реализованных мер, направленных на обеспечение работоспособности, надежности и безопасности АЭС, а также проблемы влияния сокращения численности персонала на безопасность, увеличение рабочего времени, перебои с заправкой реакторов, непрерывность цепочек поставок, перегруженность служб аварийной готовности и реагирования. В числе «постпандемических» «уроков» были озвучены: гибкость и скорость ядерных реакторов, как необходимое условие для обеспечения безопасности мировой системы энергоснабжения; особая роль атомной энергии в преодолении экономических последствий пандемии коронавируса; необходимость пересмотра способов работы на АЭС (и при их строительстве) путем внедрения инновационных методов, основанных на цифровых технологиях; необходимость пересмотра действующих правил с учетом пандемий, а также при разработке интеллектуальных процедур и систем лицензирования.

Подводя итог, следует отметить, что заявившая о себе проблема обеспечения безопасности в области использования атомной энергии в условиях пандемии не должна остаться без должного внимания как со стороны международных организаций, осуществляющих деятельность в области использования атомной энергии, так и национальных регуляторов, а также научного сообщества. Реагирование на такие серьезные вызовы, значение «уроков» которых для атомной энергетики сопоставимо с «уроками», извлеченными из ядерных инцидентов, поиск превентивных мер и разработка конкретных стратегий для регулирования отрасли после пандемии требуют солидарного подхода и объединенных усилий всех заинтересованных сторон, поэтому многостороннее сотрудничество приобретает особое значение для сдерживания пандемии и смягчения ее последствий. Что касается последствий влияния пандемии COVID-19 на дальнейшее развитие мировой энергетики, в целом, и для атомной энергетики - в частности, то их еще только предстоит оценить.

Литература и источники

1. Global Energy Review 2020: The impacts of the COVID-19 crisis on global energy demand and CO2 emissions // https://www.iea.org/reports/global-energy-review-2020.

2. IEA charts COVID-19 decline in global energy demand and emission(30 April 2020) // https://world-nuclearnews.org/Articles/Global-energy-demand-and-emissions-impacted-by-COV.

3. Global Energy Review 2020: The impacts of the COVID-19 crisis on global energy demand and CO2 emissions // https://www.iea.org/reports/global-energy-review-2020.

4. Российские АЭС нарастили производство и продажи // http://strana-rosatom.ru/2020/05/07/российские-аэс-нарастилипроизводст/

5. Росатом продолжает работу по стерилизации медицинской продукции для борьбы с коронавирусом // https://www. rosatom.ru/journalist/news/rosatom-prodolzhaet-rabotu-po-sterilizatsii-meditsinskoy-produktsii-dlya-borby-s-koronavirusom/

6. Ontario nuclear supply chain joins COVID-19 response (01 May 2020) // https://world-nuclear-news.org/Articles/Ontario-nuclearsupply-chain-joins-COVID-19-respon

7. Sustaining Safe Nuclear Operations In An Influenza Pandemic: NRC Pandemic Flu Workshop April 27, 2006 // https://www.nrc.gov/docs/ML0617/ML061740199.pdf

8. NEI White Paper: Pandemic Licensing Plan (January 2007) // https://www.nrc.gov/docs/ML0704/ML070470653.pdf

9. Industrial Safety Guidelines for Nuclear Facilities. IAEA Nuclear Energy Series NP-T-3.3. IAEA, VIENNA, 2018 // https://wwwpub.iaea.org/MTCD/Publications/PDF/PUB1774_web.pdf

10. Tobey W., Roth N. How to keep nuclear power plants operating safely during the coronavirus pandemic

11. (April 16, 2020) // https://thebulletin.org/2020/04/how-to-keep-nuclear-power-plants-operating-safely-during-the-coronaviruspandemic/ 
12. «Росатом» ввел особый режим на всех российских АЭС из-за пандемии // https://www.interfax.ru/russia/701067.

13. Глава Заречного и директор Белоярской АЭС обратились жителям по ситуации с коронавирусом // https://www.atomicenergy.ru/news/2020/03/30/102505.

14. FANR COVID-19 update // https://www.fanr.gov.ae/en/media-centre/fanr-covid-19-update.

15. Fisher M., Dixit A. IAEA Steps up Support for Nuclear Facility Operators during COVID-19 Crisis (08 Apr 2020) // https://www.iaea.org/newscenter/news/iaea-steps-up-support-for-nuclear-facility-operators-during-covid-19-crisis.

16. Сеть «Опыт эксплуатации АЭС в условиях пандемии COVID-19»// https://www.iaea.org/ru/resursy/set-opyt-ekspluatacii-aesv-usloviyah-pandemii-covid-19.

17. Nuclear regulators examine response to pandemic 20 May $2020 / / \mathrm{https}$ ://world-nuclear-news.org/Articles/Nuclear-regulatorsexamine-response-to-pandemic.

ЛИЗИКОВА МАРИНА СЕРГЕЕВНА - кандидат юридических наук, старший научный сотрудник сектора предпринимательского и корпоративного права Института государства и права PAH (lizikova_m@mail.ru).

LIZIKOVA, MARINA S. - Ph.D. in Law, Senior Research Fellow of the Business and Corporate Law Department, Institute of State and Law, Russian Academy of Sciences (lizikova_m@mail.ru).

УДК 347.778

\section{ЗАПОЛЬСКИЙ С.В., СМОРЧКОВА Л.Н. ПРАВОВОЙ РЕЖИМ НАУЧНОГО ГРАНТА: ПРОБЛЕМЫ ОПРЕДЕЛЕНИЯ И ПУТИ СОВЕРШЕНСТВОВАНИЯ}

Ключевые слова: грант, грантодатель, грантополучатель, наука, научный грант, правовой режим, конкурс, финансирование, научная деятельность, научно-исследовательские работы.

В статье, на основе результатов всестороннего анализа действующего законодательства, определены уровень и пределы наиболее оптимального правового регулирования отношений, складывающихся в процессе назначения, выдачи и использования грантов, предоставляемых для финансирования деятельности в области науки. Автором предложена концепция и структура проекта федерального закона «О научных грантах и грантовой деятельности в сфере науки», обозначены ключевые идеи, на основе которых должен формироваться правовой режим научного гранта. Отдельной главой в проекте этого федерального закона предлагается детализировать правовое регулирование предоставления грантов на осуществление научно-исследовательских, опытно-конструкторских и технологических работ, как обладающих наибольшей значимостью, популярностью, финансовой емкостью и сходством с гражданско-правовыми договорными отношениями на выполнение научно-исследовательских, опытно-конструкторских и технологических работ.

\section{ZAPOLSKY, S.V., SMORCHKOVA, L.N. LEGAL REGIME OF A SCIENTIFIC GRANT: PROBLEMS OF DEFINITION AND WAYS OF IMPROVEMENT}

Keywords: grant, grantor, grantee, science, scientific grant, legal regime, competition, financing, scientific activity, research work.

In the article, based on the results of a comprehensive analysis of the current legislation, defines the level and limits of the most optimal legal regulation of relations that develop in the process of assigning, issuing and using grants provided to finance of activities in the field of science. The author proposed the concept and structure of the draft federal law "On scientific grants and grant activities in the field of science", identified key ideas on the basis of which the legal regime of a scientific grant should be formed. A separate chapter in the draft of this federal law proposes to detail the legal regulation of the provision of grants for research, experimental design and technological work, as those having the greatest significance, popularity, financial capacity and similarity with civil law contractual relations for the implementation of research, experimental -design and technological work.

Финансирование определенных видов деятельности и проектов посредством выдачи грантов прочно вошло в отдельные сферы жизни современной России с конца прошлого века. Начало возобновления вспомогательного финансового обеспечения науки, хорошо известного в дореволюционной России [1], в постсоветской России положили зарубежные гранты, предлагавшиеся российским ученым на продолжение исследований в условиях тотального перестроечного дефицита.

Гранты, будучи по своей финансово-правовой природе безвозмездными и безвозвратными целевыми вложениями, являются очень привлекательным инструментом финансирования с учетом закрепленных в законе особенностей налогообложения по подоходному налогу физических лиц и налогу на прибыль организаций. Вероятно, именно в связи с этим обстоятельством поле выдачи целевой финансовой помощи, именуемой грантами, существенно расширилось далеко за пределы проведения научных исследований и связанных с ними работ.

В то же время, как неоднократно отмечалось и в научной литературе [2, 3, 4 и др.], а также в официальных документах [5], нормативное регулирование грантовой деятельности в российском законодательстве является фрагментарным, а правовой режим самого гранта - неопределенным.

\footnotetext{
${ }^{3}$ Статья подготовлена при финансовой поддержке РФФИ в рамках научного проекта № 18-29-15035 мк «Сравнительноправовое исследование механизма назначения, выдачи и использования грантов в целях финансирования научноисследовательских, опытно-конструкторских и технологических работ»
} 